\title{
FATORES DE RISCO PARA O CÂNCER DE PRÓSTATA: POPULAÇÃO DE UMA UNIDADE BÁSICA DE SAÚDE*
}

\author{
Rildo César Nunes Czorny ${ }^{1}$, Maria Helena Pinto², Daniele Alcalá Pompeo², Denise Bereta², Luciana Ventura \\ Cardoso $^{3}$, Dorival Marques da Silva ${ }^{4}$
}

RESUMO: O estudo objetivou identificar os fatores de risco para o câncer de próstata entre homens atendidos durante o mês de novembro de 2015, em uma Unidade Básica de Saúde do estado de São Paulo. Estudo descritivo, transversal e de abordagem quantitativa, composta por 150 usuários homens que compareceram na unidade para realização de consulta e/ou acolhimento de enfermagem. Os fatores de risco com maior relevância foram idade, nível de escolaridade, sedentarismo, uso de bebida alcoólica, ingestão de carne vermelha, leite e gorduras e Índice de Massa Corporal aumentado. O exame de rastreio mais realizado foi o Antígeno Prostático Específico, seguido do toque retal. O número de participantes que apresentou algum fator de risco para o câncer de próstata foi elevado, observou-se que a história familiar da doença motiva os homens a procurarem por medidas de prevenção, com a realização de consulta com o urologista e exames de rastreio.

DESCRITORES: Saúde do homem; Neoplasias da próstata; Fatores de risco; Atenção primária à saúde.

\section{PROSTATE CANCER RISK FACTORS: POPULATION FROM A BASIC HEALTH UNIT}

\begin{abstract}
The present investigation aimed to identify prostate cancer risk factors in men assisted in a basic health unit in the state of São Paulo in November 2015. It was a descriptive, cross-sectional and quantitative study, with a sample of 150 male users that went to the unit for a medical appointment and/or nursing care. The most relevant risk factors were age, level of education, sedentary lifestyle, consumption of alcohol, red meat, milk and fat and a high body mass index. The most used screening test was the prostatespecific antigen exam, followed by a rectal examination. The number of participants that presented at least one prostate cancer risk factor was high and that family history prompted men to seek prevention measures, such as appointments with urologists and screening exams.
\end{abstract}

DESCRIPTORS: Men's health; Prostatic neoplasms; Risk factors; Primary health care.

\section{FACTORES DE RIESGO DE CÁNCER DE PRÓSTATA: POBLACIÓN DE UN CENTRO DE SALUD}

RESUMEN: El estudio apuntó a identificar los factores de riesgo de cáncer de próstata entre hombres atendidos durante noviembre de 2015 en un Centro de Salud del Estado de São Paulo. Estudio descriptivo, transversal, de abordaje cuantitativo. Muestra integrada por 150 usuarios masculinos, que comparecieron en la unidad para realización de consulta y/o acogimiento de enfermería. Los factores de riesgo de mayor relevancia resultaron: edad, nivel de escolarización, sedentarismo, abuso de bebidas alcohólicas, ingesta de carnes rojas, leche y grasas, e Índice de Masa Corporal elevado. El examen de rastreo más realizado fue el de Antígeno Prostático Específico, seguido del tacto rectal. El número de participantes que presentó factores de riesgo de cáncer de próstata fue elevado. Se observó que la historia familiar de la enfermedad motiva a los hombres a buscar medidas preventivas, realizando consultas con el urólogo y análisis de rastreo.

DESCRIPTORES: Salud del Hombre; Neoplasias de la Próstata; Factores de Riesgo; Atención Primaria de Salud.

\footnotetext{
*Artigo extraído da dissertação intitulada: "Características Sociodemográficas e Fatores de Risco para o Câncer de Próstata na Atenção Básica". Faculdade de Medicina de São José do Rio Preto, 2017.

${ }^{1}$ Enfermeiro. Mestre em Enfermagem. Enfermeiro da Prefeitura Municipal de São José do Rio Preto. São José do Rio Preto, SP, Brasil.

${ }^{2}$ Enfermeira. Doutora em Enfermagem. Docente de Enfermagem da Faculdade de Medicina de São José do Rio Preto. São José do Rio Preto, SP, Brasil.

${ }^{3}$ Enfermeira. Doutoranda em Ciências da Saúde. Faculdade de Medicina de São José do Rio Preto. São José do Rio Preto, SP, Brasil.

${ }^{4}$ Enfermeiro. Especialista em Saúde da Família. Enfermeiro do Hospital Universitário da Universidade Federal de São Carlos. São Carlos, SP, Brasil.
} 


\section{INTRODUÇÃO}

A redução da natalidade, urbanização populacional, industrialização e avanço tecnológico têm contribuído para mudança demográfica, caracterizada pelo envelhecimento da população mundial. Juntamente com ações de promoção e recuperação da saúde, o perfil epidemiológico tem mudado, e as doenças crônico-degenerativas ultrapassam as infecciosas em incidência e mortalidade na população mundial ${ }^{(1)}$. Entre as doenças crônicas ressalta-se o câncer de próstata (CaP).

Nos últimos anos, o CaP tem sido tema de grandes debates entre os estudiosos e as instituições que são referencias no assunto. Essa patologia tem como principais fatores de risco: idade de 65 anos ou mais ( $62 \%$ dos casos diagnosticados no mundo estão nessa faixa etária), história familiar da doença, e homens da raça negra, podendo ser atribuída ao estilo de vida, e alguns alimentos vêm sendo associados a risco ou proteção. Atualmente, o tema risco e proteção relacionados ao CaP vem sendo debatido e existe esforço para que se chegue a um consenso sobre o assunto, inclusive sobre as formas de investigação da doença. Ademais, tais reflexões podem proporcionar atos eficazes em ações voltadas para o cuidado à Saúde do homem ${ }^{(2)}$.

A dificuldade dos homens em acessar a atenção primária, seja por desconhecimento, preconceito ou falta de recursos médico local, prejudica a realização do rastreamento do $\mathrm{CaP}$ e, consequentemente, impossibilita a detecção de tumores em fase inicial, prejudicando o diagnóstico e tratamento precoce ${ }^{(3)}$.

O Brasil apresenta uma das menores taxas de mortalidade por CaP na América Latina, porém, vale ressaltar que se observa uma tendência para o crescimento da doença ${ }^{(4)}$, sendo a segunda mais incidente entre os homens, com aproximadamente 1.112 .000 casos novos no mundo e significante causa de mortes na população masculina, com cerca de 307.000 mortes no ano de 2012. Para o ano de 2030 estima-se 1,7 milhão de casos novos dessa doença ${ }^{(3)}$.

Segundo estatística do Instituto Nacional do Câncer (INCA), órgão do Ministério da Saúde responsável pela prevenção e controle do câncer no Brasil, estima-se para o ano de 2017, 295.200 novos casos de câncer em homens no país, para o CaP são estimados 61.200 novos casos, ficando em primeiro lugar na distribuição proporcional dos dez tipos de câncer mais incidentes nos homens, exceto pele não melanoma com 80.850 casos novos ${ }^{(5)}$.

As estimativas do CaP para as regiões do Brasil são de 25.800 novos casos na região Sudeste, seguido das regiões Nordeste com 14.290, Sul com 13.590, Centro-Oeste com 5.050 e Norte com 2.470, e no Distrito Federal com $840^{(5)}$.

No Brasil, em 20 de setembro de 2001, foi instituído o Programa Nacional de Controle de Câncer de Próstata, que visa incluir, dentre outras, as seguintes atividades:

\footnotetext{
I - campanha institucional nos meios de comunicação, com mensagens sobre o que é o câncer de próstata e suas formas de prevenção; II - parcerias com as Secretarias Estaduais e Municipais de Saúde, colocando-se à disposição da população masculina, acima de quarenta anos, exames para a prevenção ao câncer de próstata; III - parcerias com universidades, sociedades civis organizadas e sindicatos, organizando-se debates e palestras sobre a doença e as formas de combate e prevenção a ela; IV - outros atos de procedimentos lícitos e úteis para a consecução dos objetivos desta instituição. V - sensibilizar os profissionais de saúde, capacitando-os e reciclando-os quanto a novos avanços nos campos da prevenção e da detecção precoce do câncer de próstata. Em 2014 essa lei sofreu alteração no II parágrafo do Art. 4 ${ }^{\circ}$ devido necessidade técnico-científicos ${ }^{(6: 1)}$.
}

Em 2009 o Ministério da Saúde instituiu a Política Nacional de Atenção Integral à Saúde do Homem (PNAISH), com o objetivo de promover ações voltadas ao homem, a fim de melhorar as condições da população masculina do Brasil e ampliar o acesso dos homens à atenção primária ${ }^{(7)}$.

Diante destas considerações e a campanha do novembro azul, este estudo teve como objetivo identificar os fatores de risco para o CaP entre homens atendidos durante o mês de novembro de 2015, em uma Unidade Básica de Saúde (UBS) do interior do estado de São Paulo. 
Trata-se de um estudo descritivo, do tipo transversal e de abordagem quantitativa desenvolvido em uma Unidade Básica de Saúde do interior do Estado de São Paulo, que está integrada à rede de serviços do Sistema Único de Saúde (SUS). No município onde o estudo foi realizado, existem cinco UBS que são referencias no atendimento especializado à saúde do homem. A unidade onde ocorreu o estudo atende cerca de 190 homens por mês em consulta de enfermagem.

A amostra do estudo foi composta por 150 usuários homens que compareceram na unidade para realização de consulta e ou acolhimento de enfermagem, com diversas necessidades, entre elas: renovação de receita de medicamentos para hipertensão e/ou diabetes, planejamento familiar, entrevista, coleta e orientações de exames para detecção de doenças sexualmente transmissíveis (DSTs), avaliação de curativos e orientações sobre a saúde sexual e reprodutiva.

A coleta dos dados ocorreu no mês de novembro/2015, mês em que é realizada a campanha do Novembro Azul, que visa à promoção da saúde do homem, incentivando-o a realizar exames preventivos de combate ao CaP.

Os critérios de inclusão foram: pacientes com idade igual ou maior de 18 anos, consciente e orientado. Como critérios de exclusão foram: pacientes menores de 18 anos e/ou em condições mentais prejudicadas, que impossibilitassem a entrevista.

Os dados foram obtidos por meio de entrevista com o usuário realizada no consultório de enfermagem, seguindo um instrumento de coleta de dados elaborado pelo pesquisador, que contemplava as seguintes variáveis: perfil sociodemográfico (idade, raça/cor declarada, escolaridade e renda mensal), história de CaP na família (pai e ou irmão), consulta anual com Urologista e realização de exames de rastreio para CaP (toque retal, dosagem do Antígeno Prostático Especifico - PSA, ultrassom transretal, biópsia), estilo de vida como hábitos alimentares, uso de álcool, tabaco e outras drogas inaláveis, atividade física ou sedentarismo, avaliação antropométrica e classificação do Índice de Massa Corporal (IMC), segundo a Organização Mundial de Saúde $(\mathrm{OMS})^{(8)}$.

Os dados obtidos (relatos dos participantes) foram armazenados em planilha do programa Microsoft Exce/ ${ }^{\circledast}$, de forma a possibilitar a análise estatística dos dados. Todas as variáveis foram analisadas por meio da quantidade absoluta e relativa (percentual), a fim de caracterizar a amostra do estudo.

O projeto foi aprovado pelo Comitê de Ética em Pesquisa da Faculdade de Medicina de São Jose do Rio Preto (FAMERP), Parecer $n^{\circ}$ 978.942, datado em 10 de março de 2015.

\section{RESULTADOS}

Para o alcance dos objetivos propostos e melhor compreensão dos dados obtidos, as Tabelas 1 e 2 apresentam as características sociodemográficas e os fatores de risco para o CaP na população masculina estudada.

Tabela 1 - Características sociodemográficas de uma população masculina atendida durante o mês de novembro de 2015. São José do Rio Preto, SP, Brasil, 2015

\begin{tabular}{lcc} 
Variáveis & $\mathbf{N}$ & $\mathbf{\%}$ \\
\hline Idade & 150 & 100 \\
\hline 18 a 29 anos & 22 & 14,67 \\
\hline 30 a 40 anos & 20 & 13,33 \\
\hline 41 a 49 anos & 16 & 10.67 \\
\hline 50 a 60 anos & 36 & 24 \\
\hline 61 a 80 anos & 48 & 32 \\
\hline Acima de 80 anos & 8 & 5,33 \\
\hline Cor autodeclarada & 150 & 100 \\
\hline Amarela & 3 & 2 \\
\hline
\end{tabular}




\begin{tabular}{lcc}
\hline Branca & 91 & 60,67 \\
\hline Parda & 42 & 28 \\
\hline Preta & 14 & 9,33 \\
\hline Escolaridade & 150 & 100 \\
\hline Sem escolaridade & 9 & 6 \\
\hline Fundamental & 75 & 50 \\
\hline Médio & 48 & 32 \\
\hline Superior & 18 & 12 \\
\hline Renda mensal* (salário mínimo) & 150 & 100 \\
\hline Menos de 1 & 7 & 4,66 \\
\hline 1 a 2 & 54 & 36 \\
\hline 2 a 3 & 55 & 36,67 \\
\hline Mais de 3 & 34 & 22,67
\end{tabular}

*valor do salário mínimo: R\$788.00 conforme decreto 8381/2014 publicado em Diário Oficial da União em 30/12/2014

Tabela 2 - Características de estilo de vida, fatores hereditários e fatores de risco para o câncer de próstata. São José do Rio Preto, SP, Brasil, 2015

\begin{tabular}{|c|c|c|}
\hline Variáveis & $\mathbf{N}$ & $\%$ \\
\hline Sedentarismo & 150 & 100 \\
\hline Não & 36 & 24 \\
\hline Sim & 114 & 76 \\
\hline Uso de bebida alcoólica & 150 & 100 \\
\hline Não & 66 & 44 \\
\hline Sim & 84 & 56 \\
\hline Frequência do uso de bebida alcoólica (por semana) & 84 & 100 \\
\hline Até 2 vezes & 53 & 63,1 \\
\hline Mais de 2 vezes & 31 & 36,9 \\
\hline Tabagismo e ou drogas inaláveis (maconha, cocaína e crack) & 150 & 100 \\
\hline Não & 113 & 75,33 \\
\hline Sim & 37 & 24,67 \\
\hline Ingestão de carne vermelha (cinco ou mais vezes/semana) & 150 & 100 \\
\hline Não & 17 & 11,33 \\
\hline Sim & 133 & 88,67 \\
\hline Ingestão de leite ou derivados (cinco ou mais vezes/semana) & 150 & 100 \\
\hline Não & 54 & 36 \\
\hline Sim & 96 & 64 \\
\hline Ingestão de gordura (cinco ou mais vezes/semana) & 150 & 100 \\
\hline Não & 74 & 49,33 \\
\hline Sim & 76 & 50,67 \\
\hline Índice de massa corporal & 150 & 100 \\
\hline Baixo peso & 5 & 3,33 \\
\hline Eutrófico & 49 & 32,67 \\
\hline Sobrepeso & 66 & 44 \\
\hline Obeso I & 23 & 15,33 \\
\hline Obeso II & 7 & 4,67 \\
\hline História de câncer de próstata na família & 35 & 100 \\
\hline Pai & 25 & 71,43 \\
\hline Irmão & 10 & 28,57 \\
\hline
\end{tabular}


De acordo com os resultados percentuais da Tabela 1, o predomínio foi dos pacientes com idade de 61 a 80 anos $(32 \%, n=48)$, consideram-se branco $(60,67 \%, n=91)$, com escolaridade de nível fundamental $(50 \%, \mathrm{n}=75)$, com renda mensal de 2 a 3 salários mínimos $(36,67 \%, \mathrm{n}=55)$.

Os dados da Tabela 2 mostram que os pacientes foram classificados como sedentários (76\%, $\mathrm{n}=114)$, ingere bebida alcoólica $(56 \%, n=84)$ até duas vezes na semana $(63,10 \%, n=53)$ e não apresenta o hábito de fumar ou uso de drogas inaláveis $(75,33 \%, \mathrm{n}=113)$. Em relação à alimentação, houve predomínio dos relatos de ingestão de carne vermelha $(88,67 \%, n=133)$, leite $(64 \%, n=96)$, gordura $(50,67 \%, n=76)$, cinco ou mais vezes por semana. O IMC prevalente foi de sobrepeso (44\%, $n=66$ ), seguido de normal $(32,67 \%, \mathrm{n}=49)$, e 35 pacientes relataram história familiar de CaP sendo $25(71,43 \%)$ de pai e $10(28,57 \%)$ de irmão.

De forma geral, os pacientes já se consultaram com o urologista $(52 \%, n=78)$. Dentre os principais exames concernentes à próstata, o PSA foi o que apresentou maior frequência $(56 \%, n=84)$ e os demais exames como ultrassom $(10,67 \%, n=16)$, toque retal $(32 \%, n=48)$ e biópsia $(8 \%, n=12)$ apresentaram menor ocorrência. Em relação à alimentação, a maioria dos pacientes ingere frutas $(76,67 \%, \mathrm{n}=115)$, vegetais $(82 \%, n=123)$ e leguminosas $(86 \%, n=129)$ (Tabela 3$)$.

Tabela 3 - Percentuais das variáveis relacionadas à prevenção do câncer de próstata. São José do Rio Preto, SP, Brasil, 2015

\begin{tabular}{lcc} 
Variáveis & $\mathbf{N}$ & $\mathbf{\%}$ \\
\hline Consulta com urologista anualmente & 150 & 100 \\
\hline Não & 72 & 48 \\
\hline Sim & 78 & 52 \\
\hline Realizam PSA anualmente & 150 & 100 \\
\hline Não & 66 & 44 \\
\hline Sim & 84 & 56 \\
\hline Já realizaram ultrassom abdominal/transretal & 150 & 100 \\
\hline Não & 134 & 89,33 \\
\hline Sim & 16 & 10,67 \\
\hline Realizam toque retal anualmente & 150 & 100 \\
\hline Não & 102 & 68 \\
\hline Sim & 48 & 32 \\
\hline Já realizaram biópsia da próstata & 150 & 100 \\
\hline Não & 138 & 92 \\
\hline Sim & 12 & 8 \\
\hline Ingestão de frutas (cinco ou mais vezes/semana) & 150 & 100 \\
\hline Não & 35 & 23,33 \\
\hline Sim & 115 & 76,67 \\
\hline Ingestão de vegetais (cinco ou mais vezes/semana) & 150 & 100 \\
\hline Não & 27 & 18 \\
\hline Sim & 123 & 82 \\
\hline Ingestão de leguminosas (cinco ou mais vezes/semana) & 150 & 100 \\
\hline Não & 21 & 14 \\
\hline Sim & 129 & 86 \\
\hline & & \\
\hline
\end{tabular}


Vários fatores de risco têm sido apontados como justificativa para o aumento da ocorrência do CaP. Segundo orientação da Sociedade Brasileira de Urologia, os homens devem iniciar a realização dos exames de rastreio aos 50 anos, exceto os da raça negra e com história familiar da doença, pai e ou irmão, que devem iniciar com 45 anos, sendo necessário individualizar a abordagem neste sentido ${ }^{(9)}$.

Os dados apontam que a maioria dos participantes está na idade preconizada para realização dos exames de rastreamento para $\mathrm{CaP}$, as características sociodemográficas como raça e renda não são fatores de risco mais preocupantes na população estudada, visto que a maior parte é da raça branca e as estatísticas mundiais apontam a predominância em indivíduos da raça negra ${ }^{(10)}$.

A renda mensal entre 2 a 3 salários mínimos e a prevalência de poucos anos de estudo sugere maior atenção por parte dos profissionais de saúde no momento de realizar orientações de saúde a esses usuários. Estudo enfatiza que a baixa escolaridade pode estar relacionada a um diagnóstico de câncer em estágio mais avançado e maior taxa de mortalidade, além de estar relacionado também a piores níveis de cuidado com a saúde ${ }^{(11)}$. Outros estudos destacam que os países de baixa e média renda apresentam os maiores índices de doenças crônicas não transmissíveis e as pessoas de menor escolaridade e renda são mais vulneráveis a este grupo de patologia, devido a maior exposição aos fatores de riscos, menos informação, menos acesso à saúde, impactando nas desigualdades sociais ${ }^{(12-13)}$.

Um estudo de coorte, com 13.109 homens suecos, que avaliou a associação entre atividade física, IMC e a incidência de CaP, verificou que a atividade física tende a estar ligada a um menor risco de desenvolvimento de câncer, podendo ser considerada como um fator protetor para o CaP ${ }^{(14)}$. A adesão a estilo de vida saudável, definido por praticar exercícios físicos em média de três horas ou mais por semana, não fumar e ter alimentação saudável, pode ser estratégia para reduzir significativamente a mortalidade por CaP e consequentemente reduzir a morbimortalidade por doenças cujos fatores de risco se assemelham ${ }^{(15)}$.

A respeito da saúde dos homens, vale ressaltar que, a cada três mortes de pessoas adultas, duas são de homens. Em geral, os homens vivem sete anos a menos do que as mulheres e são mais acometidos por doenças do coração, diabetes, câncer, hipercolesterolemia e hipertensão arterial ${ }^{(16)}$.

Diante deste quadro, o importante é que os profissionais da saúde fiquem atentos aos problemas gerais e específicos que podem acometer a população masculina e suas necessidades de cuidados, seguindo as políticas nacionais auxiliadoras do seu exercício profissional.

Os fatores de risco para o CaP podem ser associados às causas para as doenças cardiovasculares, visto que obesidade, falta de atividade física, hábitos alimentares pouco saudáveis ou excesso calórico são fatores de risco compartilhados para as doenças crônicas não transmissíveis. A obesidade tem sido associada a um maior risco de recorrência do CaP na fase após o tratamento ${ }^{(17)}$.

A obesidade pode ser uma hipótese na disparidade da incidência do CaP entre os homens negros e brancos não hispânicos. Acredita-se também que a obesidade é um fator de risco para o CaP tanto como o fator genético e ambiental ${ }^{(18)}$.

A etiologia para o CaP ainda necessita ser melhor esclarecida, diversos estudos tem buscado evidencias sobre a relação entre carcinogênese prostática e fatores ambientais como exposição a agrotóxicos, estilo de vida, sedentarismo e fator genético como história familiar ${ }^{(3)}$.

O histórico familiar de pai ou irmão com CaP antes dos 60 anos de idade pode aumentar de 3 a 10 vezes a possibilidade do indivíduo desenvolver a doença em relação à população em geral. Os estilos de vida compartilhados entre os membros da família e as características herdadas podem elevar a probabilidade de o homem desenvolver a doença ${ }^{(16)}$.

O fator história familiar foi relatado por 23,33\% dos participantes do presente estudo, sugerindo ser um fator que parece motivar os homens a atuarem preventivamente com realização de exames que, quando realizados de forma antecipada, podem diagnosticar a doença precocemente e aumentar a probabilidade de cura. Assim como outros fatores, tais como alcoolismo, tabagismo, consumo alimentar de gorduras e ingestão de leite podem também estar associados a esta neoplasia ${ }^{(16)}$.

Como foi possível evidenciar nos resultados, a maioria dos participantes não pratica atividade física regular, é classificado com sobrepeso e uma menor parte obeso grau I e II, além disso, mais de $50 \%$ 
consomem carne vermelha, leite ou derivados, gordura e bebida alcoólica. Isto sugere a necessidade de ações de promoção à saúde, a fim de estimular e encorajar esses usuários a melhorar o estilo de vida alimentar dentro das suas condições financeiras e a realizar atividade física regularmente, o que pode resultar na redução do IMC, portanto na qualidade de vida e saúde.

Uma revisão de literatura realizada em 2012, com dados da PubMed, ressalta que o interesse dos pesquisadores em descobrir como a dieta afeta a incidência e a progressão do CaP continua crescendo, mas assegura que está longe de se encontrar a dieta adequada. Estudos que relacionam os nutrientes ao CaP são limitados, na melhor das hipóteses, a tendências nos dados publicados sugerem que o consumo de carboidratos, gorduras saturadas e $\omega-6$ e certos suplementos vitamínicos podem promover o risco e a progressão da $\mathrm{CaP}^{(19)}$.

Por outro lado, o consumo de vegetais e ácidos graxos $\omega-3$ parece diminuir o risco e a progressão da doença. A heterogeneidade dos estudos dificulta concluir e considerar que o nutriente seja "bom" ou "ruim". No entanto, existem orientações que podem ser utilizadas no aconselhamento dos pacientes com CaP ou em risco para a doença, como consumir mais frutas e vegetais e não apenas os sucos sem fibras, porque a fibra retarda a absorção do açúcar do alimento, e minimizar a suplementação excessiva de vitaminas e minerais associado ao consumo moderado de calorias ${ }^{(19)}$.

A mesma revisão cita outros pequenos estudos que sugerem que os sobreviventes do CaP que são mais ativos e que relatam consumir dietas ricas em frutas e vegetais, baixo teor de gordura e baixo teor de carboidratos refinados têm em geral melhor qualidade de vida que aqueles que não se exercitam ${ }^{(19)}$. No presente estudo verificou-se que a maioria dos pacientes consome frutas e vegetais e é sedentário.

Alguns exames são fundamentais para auxiliar na detecção de alterações na próstata. O toque retal é procedimento de baixo custo, rápido, que possibilita avaliar o tamanho, o formato e a consistência da próstata, contudo não em sua totalidade ${ }^{(20)}$.

O PSA é outro exame utilizado na dosagem da proteína produzida pelas células da glândula prostática, o exame mede o valor do PSA no sangue, e os valores são referidos em nanograma por mililitro $(\mathrm{ng} / \mathrm{ml})$, vários estudos fundamentam a utilidade clínica desse fator prognóstico ${ }^{(21)}$. O escore de Gleason, que é a diferenciação histológica dos fragmentos tumorais biopsiado, juntamente com o valor do PSA, permite avaliar o risco e progressão do $\mathrm{CaP}^{(2)}$.

O ultrassom transretal tem possibilitado melhor definição anatômica da próstata, determinando o volume prostático, além de ser utilizado também para facilitar e orientar a realização da biópsia ${ }^{(22)}$.

Os resultados do presente estudo tiveram semelhanças com um estudo realizado com trabalhadores não docentes de uma universidade pública do estado de Minas Gerais. A maior parte dos pesquisados tinha mais de 50 anos e declarou ser brancos, apenas uma pequena parte tinha história familiar do CaP e era tabagista. Todavia, no que tange à prevenção, 48.90\% declararam ir anualmente a consulta médica para realizar a avaliação periódica, $84,80 \%$ afirmaram realizar o PSA anualmente e somente $26,10 \%$ realizavam o toque retal anualmente ${ }^{(23)}$.

O presente estudo indica que a realização do exame de toque retal é baixa quando comparada com a porcentagem de consultas anuais com o urologista, sugerindo que nem sempre na consulta com o especialista tal exame é realizado.

Um estudo que avaliou tendência de mortalidade por CaP na região centro-oeste do Brasil, concluiu que a melhora no diagnóstico para o CaP está relacionada com maior oferta de assistência à saúde e disponibilidade de recursos diagnósticos. Entretanto, a assistência à saúde, principalmente no serviço público, carece de melhorias estruturais e de acesso, para que o diagnóstico e consequentemente o tratamento para essa doença sejam realizados adequadamente e em tempo hábil ${ }^{(18)}$.

Um estudo americano sugeriu que a distância física percorrida até a unidade de saúde com acesso a consulta com urologista pode ser uma barreira para o diagnóstico precoce do $\mathrm{CaP}^{(24)}$.

Na UBS onde o estudo foi realizado, não há oferta de consultas com Urologista, sendo necessário que os usuários sejam atendidos em outras unidades do município. O estudo sugere que, mesmo não havendo oferta dessa especialidade na unidade, local do estudo, os usuários não demonstraram prejuízo na prevenção da doença, visto que $84,78 \%$ dos homens que estão na faixa etária para realizar 
os exames de rastreio para o CaP relataram passar por consulta anualmente com o urologista, a fim de realizar exames preventivos para $\mathrm{CaP}$.

O estudo apresentou como limitações a impossibilidade de comparar dois grupos em relação aos fatores de risco: com diagnóstico de $\mathrm{CaP}$ e sem o diagnóstico de $\mathrm{CaP}$ e com história familiar da doença. Outra limitação foi o delineamento, isto é, se fosse estudo de coorte poderia acompanhar se os pacientes com história familiar da doença desenvolveriam o CaP.

\section{CONCLUSÃO}

Os principais fatores para o CaP identificados por meio dos relatados dos pesquisados foram idade, escolaridade, sedentarismo, uso de bebida alcoólica, ingestão de carnes vermelha, leite e derivados, gorduras e IMC aumentado.

A consulta com o urologista foi realizada pela maior parte dos entrevistados que se apresentava na faixa etária preconizada para a realização dos exames de prevenção; o toque retal e o Ultrassom transretal foram os menos realizados; o consumo de frutas, verduras e leguminosas foram relatados pela maior parte dos entrevistados. Observou-se que a história familiar da doença motiva os homens a procurarem por medidas de prevenção, por meio da realização de consulta com o urologista e exames de rastreio.

Vale ressaltar que o enfermeiro desempenha papel fundamental na prevenção e promoção à saúde na atenção primária, sendo função privativa desta categoria a consulta de enfermagem, que é o momento que se deve realizar orientações, identificando precocemente os agravos, com o objetivo de aconselhá-los sobre os fatores de risco e medidas de prevenção referente ao CaP e outras doenças.

Espera-se com este estudo contribuir para a ampliação de discussões e futuras pesquisas acerca da atuação da enfermagem na educação em saúde em conjunto com outras ações importantes para a saúde do homem, que podem ajudar na prevenção do CaP e reduzir os altos custos do tratamento do câncer em estádios avançados.

\section{REFERÊNCIAS}

1. Facina T. Estimativa 2014: Incidência de Câncer no Brasil. Rev. bras. cancerol. [Internet] 2014;60(1) [acesso em 17 abr 2017]. Disponível: http://www.inca.gov.br/rbc/n_60/v01/pdf/11-resenha-estimativa-2014-incidencia-decancer-no-brasil.pdf.

2. Modesto AADA, de Lima RLB, D'Angelis AC, Augusto DK. Um novembro não tão azul: debatendo rastreamento de câncer de próstata e saúde do homem. Interface. (Botucatu). ahead of print Epub 23 fev 2017.

3. Zacchi SR, Amorim MHC, de Souza MAC, Miotto MHMB, Zandonade E. Associação de variáveis sociodemograficas e clínicas com o estadiamento inicial em homens com câncer de próstata. Cad. saúde colet. [Internet] 2014;22(1) [acesso em 17 abr 2017]. Disponível: http://dx.doi.org/10.1590/1414-462X201400010014.

4. da Silva JFS, Mattos IE, Aydos RD. Tendências de mortalidade por câncer de próstata nos Estados da Região Centro-Oeste do Brasil, 1980 - 2011. Rev. bras. epidemiol. [Internet] 2014;17(2) [acesso em 17 abr 2017]. Disponível: http://dx.doi.org/10.1590/1809-4503201400020009ENG.

5. Instituto Nacional de Câncer José Alencar Gomes da Silva (INCA). Estimativa/ 2016: Incidência de Câncer no Brasil. Rio de Janeiro: INCA; 2015.

6. Brasil. Lei n. 10.289, de 20 de setembro de 2001. Dispõe sobre a Instituição do Programa Nacional de Controle do Câncer de Próstata. Diário Oficial da Republica Federativa do Brasil, Brasília, 21 set. 2001. Seção 1: 1.

7. Cavalcanti JRD, Ferreira JA, Henriques AHB, Morais GSN, Trigueiro JVS, Torquato IMB. Assistência Integral a Saúde do Homem: necessidades, obstáculos e estratégias de enfrentamento. Esc. Anna Nery. [Internet] 2014;18(4) [acesso em 17 abr 2017]. Disponível: http://dx.doi.org/10.5935/1414-8145.20140089. 
8. Ministério da Saúde (BR). SISVAN - Notas Técnicas. SISVAN municipal - Estado Nutricional dos Usuários da Atenção Básica. [Internet] Rio de Janeiro: Sistema de Vigilância Alimentar Nutricional (SISVAN); 2004 [acesso em 02 abr 2016]. Disponível: http://tabnet.datasus.gov.br/cgi-win/SISVAN/CNV/notas_sisvan.html.

9. Sociedade Brasileira de Urologia. Nota Oficial - Rastreamento do Câncer de Próstata. [Internet] Rio de Janeiro: Sociedade Brasileira de Urologia; 2016 [acesso em 07 dez 2016]. Disponível: http://portaldaurologia.org.br/ noticias/nota-oficial-rastreamento-do-cancer-de-prostata-2/.

10. Romero FR, Romero AW, de Almeida RMS, Tambara Filho R. The prevalence of prostate cancer in Brazil is higher in Black men than in White men: systematic review and meta-analysis. Int. braz j urol. [Internet] 2012;38(4) [acesso em 17 abr 2017]. Disponível: http://dx.doi.org/10.1590/S1677-55382012000400002.

11. Souza RS, Simão DAS, Lima EDRP. Perfil sociodemográfico e clínico de pacientes atendidos em um serviço ambulatorial de quimioterapia paliativa em Belo Horizonte. REME rev. min. enferm. [Internet] 2012;16(1) [acesso em 17 abr 2017]. Disponível: http://www.reme.org.br/artigo/detalhes/498.

12. World Health Organization (WHO). Global status report on non communicable diseases 2010. Geneva: WHO; 2011.

13. Malta DC, da Silva Junior JB. O Plano de Ações Estratégicas para o Enfrentamento das Doenças Crônicas Não Transmissíveis no Brasil e a definição das metas globais para o enfrentamento dessas doenças até 2025: uma revisão. Epidemiol. Serv. Saúde. [Internet] 2013;22(1) [acesso em 17 abr 2017]. Disponível: http://dx.doi. org/10.5123/S1679-49742013000100016.

14. Grotta A, Bottai M, Adami HO, Adams SA, Akre O, Blair SN, et al. Physical activity and body mass index as predictors of prostate cancer risk. World J Urol. [Internet] 2015;33(10) [acesso em 17 abr 2017]. Disponível: http:// dx.doi.org/10.1007/s00345-014-1464-5.

15. Kenfield SA, Batista JL, Jahn JL, Downer MK, Blarigan ELV, Sesso HD, et al. Development and application of a lifestyle score for prevention of lethal prostate cancer. J Natl Cancer Inst. [Internet] 2015;108(3) [acesso em $17 \mathrm{abr}$ 2017]. Disponível: http://dx.doi.org/10.1093/jnci/djv329.

16. de Medeiros AP, de Menezes MFB, Napoleão AA. Fatores de risco e medidas de prevenção do câncer de próstata: subsídios para a enfermagem. Rev. bras. enferm. [Internet] 2011;64(2) [acesso em 17 abr 2017]. Disponível: http://dx.doi.org/10.1590/S0034-71672011000200027.

17. Moyad MA. Preventing aggressive prostate cancer with proven cardiovascular disease preventive methods. Asian J Androl. [Internet] 2015;17(6) [acesso em 17 abr 2017]. Disponível: https://dx.doi.org/10.4103\%2F1008682X.156854.

18. Barrington WE, Schenk JM, Etzioni R, Arnold KB, Neuhouser ML, Thompson Junior IM, et al. Difference in association of obesity with prostate cancer risk between US African American and non-hispanic white men in the selenium and vitamin e cancer prevention trial (SELECT). JAMA Oncol. [Internet] 2015;1(3) [acesso em $17 \mathrm{abr}$ 2017]. Disponível: https://doi.org/10.1001/jamaoncol.2015.0513.

19. Masko EM, Allott EH, Freedland SJ. The relationship between nutrition and prostate cancer: is more always better?. Eur Urol. [Internet] 2013;63(5) [acesso em 17 abr 2017]. Disponível: https://doi.org/10.1016/j. eururo.2012.11.012.

20. de Souza LM, Silva MP, Pinheiro IS. Um toque na masculinidade: a prevenção do câncer de próstata em gaúchos tradicionalistas. Rev. Gaúcha Enferm. [Internet] 2011;32(1) [acesso em 17 abr 2017]. Disponível: http:// dx.doi.org/10.1590/S1983-14472011000100020.

21. Migowski A, Azevedo e Silva G. Sobrevida e fatores prognósticos de pacientes com câncer de próstata clinicamente localizado. Rev. Saúde Pública. [Internet] 2010;44(2) [acesso em 17 abr 2017]. Disponível: http:// dx.doi.org/10.1590/S0034-89102010000200016.

22. Mafra RSCP, Fernandes FL, Batalha AXF, Carneiro PS, Veloso DFM, et al. Precisão da estimativa do peso prostático por ultrassonografia transretal e exame de toque retal em comparação ao peso real da próstata após prostatectomia radical. Arq. Catarin. Med. [Internet] 2013;42(3) [acesso em 17 abr 2017]. Disponível: http://www. acm.org.br/revista/pdf/artigos/1238.pdf.

23. Gomes CRG, Izidoro LCR, da Mata LRF. Risk factors for prostate cancer, and motivational and hindering 
aspects in conducting preventive practices. Invest. Educ. Enferm. [Internet] 2015;33(3) [acesso em 17 abr 2017]. Disponível: http://dx.doi.org/10.17533/udea.iee.v33n3a04.

24. Holmes JA, Carpenter WR, Wu Y, Hendrix LH, Peacock S, Massing M, et al. Impact of distance to a urologist on early diagnosis of prostate cancer among black and white patients. J. Urol. [Internet] 2012;187(3) [acesso em 17 abr 2017]. Disponível: https://doi.org/10.1016/j.juro.2011.10.156. 